\title{
Surgical Outcome of Giant Vestibular Schwannomas: A Retrospective Analysis
}

\author{
Mohamed I. Refaat ${ }^{1, \odot}$ Omar Y. Abdallah ${ }^{1, \odot}$ \\ ${ }^{1}$ Department of Neurosurgery, School of Medicine, Cairo University, \\ Giza Governorate, Egypt \\ Address for correspondence Mohamed I. Refaat, PhD, Department \\ of Neurosurgery, School of Medicine, Cairo University, Giza \\ Governorate 12613, Egypt (e-mail: mrefat_77@yahoo.co.uk).
}

Indian J Neurosurg 2021;10:190-193.

\begin{abstract}
Background Giant vestibular schwannomas, more than $4.5 \mathrm{~cm}$ in diameter, are still representing the majority of vestibular schwannomas in developing countries. The philosophy of incomplete and intracapsular tumor excision has been introduced in the management of these giant tumors, balancing the long-term tumor control and postoperative clinical outcome.

Objective The aim was to review the cases with giant vestibular schwannomas and studying their prevalence, morbidity, and mortality rates.

Patients and Methods This study was conducted retrospectively on data of patients who had undergone microsurgical excision of vestibular schwannomas in our institute between January and December 2017. The functional outcome of the patients was assessed by comparing the preoperative and the postoperative neurological examination, as well as the Karnofsky performance score.

Results Twenty-two cases with vestibular schwannomas were included in this study.

\section{Keywords}

- giant vestibular schwannomas

- facial nerve preservation

- bulbar affection

- retrosigmoid approach

- cerebellopontine angle

Among these 22 cases, 15 cases had giant vestibular schwannomas (68.2\%). The tumors' largest extracanalicular diameters ranged from $4.5 \mathrm{~cm}$ to $6.2 \mathrm{~cm}$. Postoperative images showed gross total excision of the tumor in 11 cases (73.3\%), and subtotal excisions in four cases (26.7\%). Twelve cases $(80 \%)$ had postoperative facial palsy. We encountered no mortality in our cases and three cases (20\%) were symptom-free postoperatively (apart from hearing affection).

Conclusion Large and giant vestibular schwannomas are still commonly met in neurosurgical practice in developing countries; they have different behaviors and presentations from those of smaller tumors. Both patient and surgeon expectations from surgery should be toward no mortality and mild or no morbidities.
\end{abstract}

\section{Introduction}

Vestibular schwannomas (VSs) are benign, slowly growing tumors arising from the inferior vestibular nerve. They are the most common tumors of the cerebellopontine angle region. ${ }^{1}$ Excision of VSs will always remain a surgical challenge for all neurosurgeons; this is due to their proximity not only to the VII-VIII nerve complex, but also to the brain stem. Large-sized VSs may be extending downward to reach the lower cranial nerves, or upward to reach the trigeminal nerve. ${ }^{2}$

published online

October 26, 2021
DOI https://doi.org/ $10.1055 / \mathrm{s}-0039-3402930$ ISSN 2277-954X.
In the past years, patients' expectations from VS surgery have risen from preservation of life to preservation of facial functions and even to preservation of hearing. ${ }^{3}$ Recent studies have documented mortality rates as low as $0.4 \%$ and anatomical preservation of facial nerve approaching $100 \%$. These results can be achieved with small-sized tumors; however, this remains doubtful with large and giant VSs. ${ }^{4}$

Many published research works are discussing the largesized VS, with introduction of the term giant VS to describe tumors exceeding 4 or $4.5 \mathrm{~cm}$ in maximum diameter. ${ }^{5}$ With

(C) 2021. Neurological Surgeons' Society of India.

This is an open access article published by Thieme under the terms of the Creative Commons Attribution-NonDerivative-NonCommercial-License, permitting copying and reproduction so long as the original work is given appropriate credit. Contents may not be used for commercial purposes, or adapted, remixed, transformed or built upon. (https://creativecommons.org/licenses/by-nc-nd/4.0/).

Thieme Medical and Scientific Publishers Pvt. Ltd. A-12, 2nd Floor, Sector 2, Noida-201301 UP, India 
the higher availability of neuroimaging, the prevalence of these giant VSs is declining over the past years, representing $2 \%$ of all VSs in Western countries. However, in less developed countries they are still common and represent a majority of VSs (as high as $50 \%$ in India). ${ }^{6,7}$

The clinical presentation of giant VSs differs from that of small-sized tumors. These giant tumors can present with manifestations of hydrocephalus, brain stem compression, facial palsy, bulbar cranial nerves' symptoms, besides loss of hearing. Surgery for giant VSs is more challenging, with higher morbidity and mortality rates than with surgeries of smaller tumors. ${ }^{8}$ The philosophy of incomplete and intracapsular tumor excision has been introduced in the management of giant VSs, balancing the long-term tumor control and the preservation of the facial nerve. ${ }^{9}$

This research was conducted to review cases with giant VSs presenting to our institution, study their prevalence among all VSs, analyze the morbidity and mortality rates of these tumors, and determine the feasibility of seeking total tumor excision.

\section{Patients and Methods}

This study was conducted retrospectively on data of patients who had undergone microsurgical excision of VSs in the neurosurgery department of Cairo University hospitals in the period between January and December 2017. We studied the data of cases with all tumor sizes as well as cases with giant VSs. For the purposes of the study, we classified giant VSs as those tumors having largest extracanalicular diameter of $4.5 \mathrm{~cm}$ or more. Patients with bilateral tumors "neurofibromatosis type II" and recurrent were not included in the studied population.

The patients' pre and postoperative clinical examination was reviewed from the records. Assessment of hearing was conducted using pure tone audiometry and the facial nerve function was divided to either normal, incomplete loss, or complete loss. The pre and postoperative images were reviewed. The maximum extracanalicular tumors' diameters were measured in the MRI. Postoperative images were reviewed for detection of degrees of tumor excision. Gross total excision was considered if more than $90 \%$ of tumor volume was excised, while subtotal excision was considered for less than $90 \%$ removal.

Operative details: All cases were operated upon in parkbench position with the head fixed using the three-pin Mayfield skull clamp. The standard retrosigmoid approach was utilized in all cases. Cases presenting with preoperative hydrocephalus had a contralateral frontal ventriculoperitoneal shunt inserted a few days before attacking the tumor; otherwise, lumbar drains were used to drain cerebrospinal fluid (CSF) after opening the dura to aid in retraction and reduce intracranial pressure. Facial nerve monitoring and the cavitron ultrasonic aspirator were used in some cases. The functional outcome of the patients was assessed by comparing the preoperative and the postoperative neurological examination, as well as the Karnofsky performance scale (KPS). ${ }^{10}$

\section{Results}

Twenty-two cases with VSs were surgically treated is our department during the study period. Among these 22 cases, 15 cases had giant VSs. This represented $68.2 \%$ of all VSs cases treated in our department. Patient demography: The age of the cases ranged from 18 to 62 years, mean age was 34 years, and the standard deviation was 13.2. There was a slight female predominance among cases included in this study as there were 10 females (66.7\%) and 5 males (33.3\%).

Preoperative clinical condition: As expected, all cases included in this study were afflicted with hearing loss to variable degrees. As detected by pure tone audiometry, eight cases $(53.3 \%)$ had profound sensory neural hearing loss (SNHL), five cases (33.3\%) had moderate SNHL, while two cases (13.3\%) had mild SNHL. Eight cases (53.3\%) had preoperative signs of cerebellar ataxia and gait imbalance. Bulbar symptoms were noticed in two cases (13.3\%) and their direct laryngoscopy detected ipsilateral vocal cord paralysis. Three cases (20\%) had trigeminal nerve affection. Preoperative facial palsy was noticed in four cases (26.7\%), one case had complete facial palsy, less than III on the House-Brackmann scale, ${ }^{11}$ and the remaining three cases had some degrees of facial paresis, more than III on House-Brackmann scale. Six cases (40\%) presented with signs of acute hydrocephalus, and these cases were operated upon by insertion of contralateral frontal ventriculoperitoneal shunt a few days before surgery. Tumor sizes: the tumors largest extracanalicular diameters ranged from 4.5 to $6.2 \mathrm{~cm}$. The mean size was $5.14 \mathrm{~cm}$ and the standard deviation was 0.56 .

Surgical details: All cases were operated by the retrosigmoid approach. Six cases had a ventriculoperitoneal shunt inserted a few days before direct tumor attack. The Cavitron ultrasonic aspirator was available in eight surgeries, while neurophysiological monitoring was used for identification and protection of the facial nerve in seven cases. Intraoperative anatomical preservation of the facial nerve was achieved in 11 out of the 15 cases. Postoperative images showed gross total excision of the tumor in 11 cases (73.3\%), and subtotal excisions in four cases (26.7\%). The diagnosis of VS was histopathologically confirmed postoperatively in all cases.

General outcome: The median preoperative Karnofsky scale was 78.6, ranging from 60 to 90 . While the median postoperative Karnofsky scale was 79.3, ranging from 50 to 90. Follow-up and recurrences: The average duration for follow-up in our study was 12 months, ranging from 7 to 14 months. Given the fact that this was a short period for follow-up, we did not meet any tumor recurrences or further regrowth of residual tumors.

Clinical outcome and complications: None of the cases showed postoperative improvement in hearing functions, as 13 cases $(86.7 \%$ ) had postoperative profound SNHL, while two cases $(13.3 \%)$ had moderate SNHL. Signs of cerebellar ataxia and gait imbalance showed postoperative improvement in five out of the eight cases who suffered from preoperative ataxia (62.5\%), while new onset of ataxia was noticed in two cases (13.3\%). Postoperative improvement of the bulbar symptoms was noticed in one of the two cases that 
had preoperative bulbar affection and was weaned from the tracheostomy tube gradually. No new cases suffered from postoperative bulbar affection. One case suffered from postoperative hemiplegia related to a small brainstem infarction. Twelve cases (80\%) had postoperative facial palsy, which on the House-Brackmann scale was less than III in 5 cases (33.3\%), and more than III in 7 cases (46.7\%).

We encountered no mortalities in our cases and three cases $(20 \%)$ were symptom-free postoperatively (apart from hearing affection). The mean hospital stay for our cases was 12.5 days (range from 5 to 26 days).

\section{Discussion}

Giant VSs, more than $4.5 \mathrm{~cm}$ in diameter, are still representing the higher percentage of VSs in developing countries due to several reasons. Among these reasons are included the unavailability of MRI in rural areas, lack of screening clinical examinations, and patients ignoring minor complaints, especially hearing troubles. Most of the published VS case series are lacking a focus on giant tumors, their natural history, and prognosis. It is evident that surgery for a giant tumor will be more difficult than surgery for a small one, and it harbors a higher incidence of postoperative morbidities and mortality.

We investigated cases operated upon for VSs in our institute over a year: $68.2 \%$ of cases were giant-sized (more than 4.5 maximal diameter). The mean tumor's diameter was $5.14 \mathrm{~cm}$, ranging from to 4.5 to $6.2 \mathrm{~cm}$. We noticed a slight female predominance among cases included in the study (66.7\% of cases). The age of the cases ranged from 18 to 62 years (mean age was 34 years).

The study published by Pai et al was conducted on cases managed over 22 years. They included cases with tumors more than $3.5 \mathrm{~cm}$ in diameter. They reported that large and giant tumors were $7.5 \%$ of all VSs operated in their center. They also reported female predominance and the mean age was 43 years. The mean tumor size of cases included in the study was $4.1 \mathrm{~cm} .{ }^{12}$ The huge difference between the incidence of giant tumors in this study and our study is evident, and is explained by the fact that this study was conducted in Europe, where there is early tumor detection and high-availability of diagnostic tools and patient screening. However, when we compared our results with other studies coming from the East, we found higher incidence of giant tumors matching with our results. A majority (97.3\%) of cases in the study published by Jain et al had giant and large VSs. ${ }^{7}$ A considerable percentage (56.2\%) of cases included in the study published by Turel et al had tumors larger than $4 \mathrm{~cm}$ in diameter. ${ }^{5}$ These studies were conducted in two Indian neurosurgical institutes. ${ }^{5,7}$

When analyzing the data collected from these cases with giant VSs, we found that all cases suffered from hearing loss, which was profound SNHL in more than half of the cases. Also, more than half of the cases had signs of cerebellar ataxia preoperatively. Preoperative facial palsy was detected in $26.7 \%$ of the cases, while $40 \%$ of cases presented with signs of hydrocephalus preoperatively.
This preoperative clinical presentation matches with studies coming from developing countries discussing giant and large tumors, unlike other Western studies where most of the patients are diagnosed early with smaller tumors, and hence the usual presentations are tinnitus and mild hearing loss. 5.7

The retrosigmoid approach was utilized for all cases. Early detection of the facial nerve medially was targeted after intracapsular debulking of the tumors, aiming for the anatomical preservation of the facial nerve, which was achieved in $73 \%$ of cases. Small adherent parts of the tumors related to the brainstem or the facial nerve were not attempted to be removed.

Gross total excision was achieved in $73 \%$ of cases, while the remaining $27 \%$ had subtotal excisions. As expected, none of the cases showed any postoperative improvement in their hearing; however, signs of cerebellar affection improved in $62.5 \%$ of affected cases. A significant percentage $(80 \%)$ of cases had postoperative facial palsy, which was complete in $33.3 \%$ of cases, and incomplete in $46.7 \%$. We encountered no mortalities in our cases and three cases (20\%) were symptom-free postoperatively (apart from hearing affection).

These results were not far beyond published Western research works with excellent rates of total excision in giant VSs (95-100\%). The main difference was the functional facial nerve outcomes. They reported a very good facial nerve outcome in 70 to $70 \%$ of cases, which is very far from our results. ${ }^{5,8,13,14}$

Our results cleared the point that safe total removal of giant VSs could be achieved, without severe morbidities or mortalities. The main surgical challenge is to achieve the patients' expectations from surgery, particularly concerning the facial nerve functions. This can be achieved by increasing surgical training and skills and also through the wider availability of modern tools such as the Cavitron ultrasonic aspirator, intraoperative facial monitoring, as well as modern MRI scans that can detect the exact position of the facial nerve in relation to the tumor preoperatively.

\section{Conclusions}

Large and giant VSs are still commonly met in neurosurgical practice in developing countries, as they have different behaviors and presentations from those of smaller tumors. Both patient and surgeon expectations from surgery should be toward no mortality and mild or no morbidities. Facial nerve preservation should be targeted and this can be achieved using intraoperative neurophysiologic monitoring and the utilization of the ultrasonic aspirators as well as intracapsular excisions. More care should be given to patient education and diagnosis of these tumors at an early stage when they are small in size.

\section{Ethical Approval}

This study was a retrospective study; the need for ethical committee approval was waived by the local ethical committee of the neurosurgery department of Cairo University, Egypt. 


\section{Note}

The datasets used and/or analyzed during the current study are available from the corresponding author on reasonable request.

\section{Authors' Contributions}

Both authors contributed to the collection of data, analysis, and writing of the manuscript. Surgeries were performed in the neurosurgery department of Cairo University hospitals, Egypt.

\section{Funding}

This study was self-funded by the authors.

\section{Conflict of Interest}

None declared.

\section{References}

1 Harati A, Scheufler KM, Schultheiss R, et al. Clinical features, microsurgical treatment, and outcome of vestibular schwannoma with brainstem compression. Surg Neurol Int 2017;8:45

2 Zhao F, Wang B, Yang Z, et al. Surgical treatment of large vestibular schwannomas in patients with neurofibromatosis type 2: outcomes on facial nerve function and hearing preservation. J Neurooncol 2018;138(2):417-424

3 Cheng S, Naidoo Y, da Cruz M, Dexter M. Quality of life in postoperative vestibular schwannoma patients. Laryngoscope 2009;119(11):2252-2257
4 Ansari SF, Terry C, Cohen-Gadol AA. Surgery for vestibular schwannomas: a systematic review of complications by approach. Neurosurg Focus 2012;33(3):E14

5 Turel MK, D'Souza WP, Chacko AG, Rajshekhar V. Giant vestibular schwannomas: surgical nuances influencing outcome in 179 patients. Neurol India 2016;64(3):478-484

6 Gerganov VM, Samii M. Giant vestibular schwannomas. World Neurosurg 2012;77(5-6):627-628

7 Jain VK, Mehrotra N, Sahu RN, Behari S, Banerji D, Chhabra DK. Surgery of vestibular schwannomas: an institutional experience. Neurol India 2005;53(1):41-45, discussion 45

8 Samii M, Gerganov VM, Samii A. Functional outcome after complete surgical removal of giant vestibular schwannomas. J Neurosurg 2010;112(4):860-867

9 Zhang S, Liu W, Hui X, You C. Surgical treatment of giant vestibular schwannomas: facial nerve outcome and tumor control. World Neurosurg 2016;94:137-144

10 Karnofsky DA, Burchenal JH. The clinical evaluation of chemotherapeutic agents in cancer. In MacLeod CM, ed, Evaluation of Chemotherapeutic Agents. Columbia Univ press; 1949:196

11 House JW, Brackmann DE. Facial nerve grading system. Otolaryngol Head Neck Surg 1985;93(2):146-147

12 Pai I, Bowman J, Thomas N, et al. Management of large and giant vestibular schwannomas. Skull Base 2011;21(6):379-384

13 Silva J, Cerejo A, Duarte F, Silveira F, Vaz R. Surgical removal of giant acoustic neuromas. World Neurosurg 2012;77(5-6):731-735

14 Charpiot A, Tringali S, Zaouche S, Ferber-Viart C, Dubreuil C. Perioperative complications after translabyrinthine removal of large or giant vestibular schwannoma: outcomes for 123 patients. Acta Otolaryngol 2010;130(11):1249-1255 